\title{
Zero-knowledge universal lossless data compression
}

\author{
Rodolfo A. Fiorini* \\ Politecnico di Milano University, 20133 Milano, Italy
}

\begin{abstract}
Advanced instrumentation, dealing with nanoscale technology at the current edge of human scientific enquiry, like X-Ray CT, generates an enormous quantity of data from single experiment. The very best modern lossless data compression algorithms use standard approaches and are unable to match high end requirements for mission critical application with full information conservation (a few pixels may vary by com/decom processing). In previous papers published elsewhere, we have already shown that traditional $\boldsymbol{Q}$ Arithmetic can be regarded as a highly sophisticated open logic, powerful and flexible bidirectional formal language of languages, according to "Computational Information Conservation Theory" (CICT). This new awareness can offer competitive approach to guide more convenient algorithm development and application for combinatorial lossless compression. To achieve true lossless com/decom and to overcome traditional constraints, the universal modular arithmetic approach, based on CICT Solid Number (SN) concept, is presented. To check practical implementation performance and effectiveness, an example on computational imaging is benchmarked by key performance index and compared to standard well-known lossless compression techniques. Results are critically discussed.
\end{abstract}

\section{Introduction}

Advanced instrumentation, dealing with nanoscale technology at the current edge of human scientific enquiry, like X-Ray CT, generates an enormous quantity of data from single experiment [1]. Even in MicroCT or Discrete Tomography (DT) by electron microscopy, 2-D projection images are acquired from various angles, by tilting the sample, generating new challenges associated with the problem of formation, acquisition, compression, transmission, and analysis of an enormous quantity of data [2], [3]. During this time of exploding data growth, disk manufacturers have begun running into the physical limitations of current storage technology (e.g., disk platter bit density, data transfer, etc.) and to seek new technologies to rely on [4].

Contemporary approaches to data compression vary in time delay or impact on application performance as well as in the amount of compression and loss of data. Two approaches that focus on data loss are lossless (no data loss) and lossy (some data loss for higher compression ratio). Different from natural images, advanced technology imaging and medical images generally have two special issues that should be noted in compression. First, they are sensitive to compression errors. Large distortion arising from lossy compression may invalidate their interpretation and diagnostic values. Second, especially the monochromatic images usually have extended dynamic range. Each pixel typically contains 16 or 12 bits per channel, compared to the 8-bitdepth pixels of common natural images. Nevertheless, both lossless and lossy compression schemes have been proposed to compression image application. Lossy compression (irreversible, i.e. information dissipation) schemes can achieve much higher Compression Ratio (CR) than lossless ones, by allowing some distortion in reconstruction. However, for mission critical application, the lossy distortion level must have to be supervised by qualified experts to avoid possible legal and diagnostic problems, especially for medical application [5],[6].

Lossless compression (reversible, i.e. information conservation) schemes provide reconstructed images identical to the original ones, but suffer from relative lower compression ratio, typically between $2: 1$ and $4: 1$. Furthermore, a single compression ratio (CR) cannot serve as a guideline for the compression of medical images, as compression artifacts vary considerably with image content, scanning technique, and compression algorithm [7]. Some image compression schemes have been adopted by the DICOM standard [8], among which JPEG 2000 [9]. It is based on a reversible integer wavelet transform and it is considered to have the best performance and a few good properties. JPEG 2000 provides both lossy and lossless compressions for images with bit depth no more than 16 bit per channel, and allows progressive transmission with SNR (Signal to Noise Ratio) and spatial scalability. In particular, it supports ROI (Region Of Interest) coding, so that image regions with diagnostic importance can have much higher quality than the other parts.

The codestream obtained after compression of an image with JPEG 2000 is downscalable in nature, meaning that it can be decoded in a number of ways; for instance, by truncating the codestream at any point, one may obtain a representation of the image at a lower resolution, or signal-to-noise ratio. However, as a

*Corresponding author: rodolfo.fiorini@polimi.it

C) The Authors, published by EDP Sciences. This is an open access article distributed under the terms of the Creative Commons Attribution License 4.0 (http://creativecommons.org/licenses/by/4.0/). 
consequence of this flexibility, JPEG 2000 requires encoders/decoders that are complex and computationally demanding. JPEG 2000 has been published as an ISO standard, ISO/IEC 15444. According to Wikipedia, as of 2013, JPEG 2000 is not widely supported in web browsers, and hence is not generally used on the Internet [10]. Traditional lossless compression techniques can be mapped to three main reference areas according to their compression underlining principle: a) Entropy encoding; b) Dictionary; c) Others. The very best modern lossless compressors use probabilistic models, such as prediction by partial matching.

\section{Contemporary techniques limitations}

As a matter of fact, we can say that almost all contemporary data compression techniques are still based on binary code uncertainty probabilistic evaluation, by treating messages to be encoded as a sequence of independent and identically distributed random variables, according to the probabilistic approach of the father of probabilistic communication theory [11]. Their major points of weakness for contemporary data compression techniques to be used for high demanding lossless applications are:

1) context and data type compression effectiveness and efficiency strong dependence for algorithm optimization;

2) centralized data size compression/decompression speed and space limitations;

3) fixed bit depth for image com/decom processing or just downgrading.

Third point is especially inadequate for discrete tomography and advanced biomedical imaging applications where high data reliability is required, at different bit depth output representation scenarios (for instance, a network with many different output devices with different output bit depth each).

Our main interest is most focused on convenient zero-knowledge universal lossless comp/decomp algorithm for advanced applications as discrete tomography, computed tomography and medical images with true "Arbitrary Bit Depth" (ABD) resolution [5],[6]. No contemporary lossless comp/decomp algorithm is able to satisfy this kind of specification yet. Furthermore it would be a plus to use traditional BD (Bit Depth) settings of images, more efficiently, so that only a small part of the coded bit-stream is needed at the decoder to lossless regenerate and display the original image, with recommended BD parameters for specific need.

\section{Combinatorial lossless compression}

Human biological transducers, by which we acquire information on the outer world interacting with it, are intrinsically discrete. This means that our perception of continuous shapes is just an illusion created by our mind. From this ground we can infer that an illusion of continuity can be achieved by discrete optimized support, without even noticing any difference, maintaining thus a maximum representation coverage property.

In general, optimization problems can be divided into two large categories depending on whether the variables are continuous or discrete. In solving a classical linear optimization problem (continuous), one can exploit the fact that, due to the convexity of the feasible region, any locally optimal solution is a global optimum.

An optimization problem with discrete variables is known as a combinatorial optimization problem. In a combinatorial optimization problem, we are looking for an object such as an integer, permutation or graph from a finite (or possibly countable infinite) set [12]. In many such problems, exhaustive search is not feasible. It operates on the domain of those optimization problems, in which the set of feasible solutions is discrete or can be reduced to discrete, and in which the goal is to find the best solution. In finding global optima for integer optimization problems, one is required to prove that a particular solution dominates all others by arguments, other than the calculus-based approaches of convex optimization.

While in linear optimization problems, the feasible region is convex, the feasible regions of classical integer optimization problems consists of either a discrete set of points or, in the case of general MILP (Mixed Integer Optimization Problem), a set of disjoint convex polyhedra [13]. Some common problems involving combinatorial optimization are the Traveling Salesman Problem (TSP) and the minimum spanning tree problem. TSP is NP-hard problem in combinatorial optimization, notoriously, and $\mathrm{P}$ versus NP problem is a major unsolved problem in computer science [14]. Nevertheless, when a discrete optimized solution is obtained, the discrete approach reveals to be, in this sense, highly convenient because it strongly decreases the computational cost and the complexity of the system for representation modelling. According to computational information conservation theory (CICT) [15],[16], in Arithmetic the structure of closure spaces (across an Outer-Inner Universe boundary) is self-defined by Natural Numbers Reciprocal Space (RS) representation [17],[18]. By this way, Natural Number can be thought as both structured object and symbol at the same time.

\subsection{Linear arithmetic closure}

As a simple example, let us consider a generic fraction $\mathrm{N} / \mathrm{D}$, where $\mathrm{N}$ and $\mathrm{D} \in \boldsymbol{Z}$, and $\mathrm{D}=\overline{0} 7 . \overline{0}, \mathrm{~L}$ an integer counter, $\mathrm{N}$ the dividend, $\mathrm{Q}$ the quotients and $\mathrm{R}$ the remainders of the long hand division. In traditional arithmetic long division algorithm (the one you learn to divide at elementary school), usual dominant result (quotient, Q) is important, and minority components (remainders, R) are always discarded. We can write the L, N, Q and R sequences as from Table 1.

Table 1. L, N, Q, R SEQUENCES.

$\begin{array}{rrrrrrrrrrrrrrrr}\mathbf{L} & 1 & 2 & 3 & 4 & 5 & 6 & 7 & 8 & 9 & 10 & 11 & 12 & 13 & 14 & 15 \ldots \\ \mathbf{N} & 10 & 20 & 30 & 40 & 50 & 60 & 70 & 80 & 90 & 100 & 110 & 120 & 130 & 140 & 150 \ldots \\ \mathbf{Q} & 1 & 2 & 4 & 5 & 7 & 8 & 10 & 11 & 12 & 14 & 15 & 17 & 18 & 20 & 21 \ldots \\ \mathbf{R} & 3 & 6 & 2 & 5 & 1 & 4 & 0 & 3 & 6 & 2 & 5 & 1 & 4 & 0 & 3 \ldots\end{array}$


for $\mathrm{L}=1,2,3, \ldots$

In this specific case, we see that the remainder $\mathbf{R}$ sequence repeats itself after 7 remainders with $\mathrm{R}(\mathrm{L})$ given by:

$\mathrm{R}(1+7 \mathrm{n})=\mathrm{R} 1=3, \mathrm{R}(2+7 \mathrm{n})=\mathrm{R} 2=6, \mathrm{R}(3+7 \mathrm{n})=\mathrm{R} 3=2$, $\mathrm{R}(4+7 \mathrm{n})=\mathrm{R} 4=5, \mathrm{R}(5+7 \mathrm{n})=\mathrm{R} 5=1, \mathrm{R}(6+7 \mathrm{n})=\mathrm{R} 6=4$, $\mathrm{R}(7+7 \mathrm{n})=\mathrm{R} 7=0$ for $\mathrm{n}=0,1,2,3, \ldots$

Accordingly, we can rearrange the quotients with respect to their cyclic remainder value respectively, obtaining the R1, R2, R3, R4, R5, R6, R7 sequences as from Table 2 .

Table 2. R1, R2, R3, R4, R5. R6, R7 SEQUENCES.

R1 Q1，Q8，Q15，Q22，Q29，Q36，Q43，Q50,.. R2 Q2, Q9, Q16, Q23, Q30, Q37, Q44, Q51,... R3 Q3, Q10, Q17, Q24, Q31, Q38, Q45, Q52,... R4 Q4, Q11, Q18, Q25, Q32, Q39, Q46, Q53,.. R5 Q5, Q12, Q19, Q26, Q33, Q40, Q47, Q54,.. R6 Q6, Q13, Q20, Q27, Q34, Q41, Q48, Q55,... R7 Q7, Q14, Q21, Q28, Q35, Q42, Q49, Q56,..

In a more compact modular format, we can write:

$\mathrm{R}(\mathrm{L})=3 \mathrm{~L} \bmod (07)$ and

$\mathrm{D} Q(\mathrm{~L})=\mathrm{N}-\mathrm{R}(\mathrm{L})$, where $\mathrm{N}=10 \mathrm{~L}$.

We can interpret the remainders $R(L)$ as the linear (unfolded) arithmetic closure to $\mathrm{N}$ with respect to $\mathrm{D}$ $\mathrm{Q}(\mathrm{L})$.

\subsection{Exponential rational closure}

For sake of simplicity, at elementary level, let us consider fraction $1 / \mathrm{D}$, where $\mathrm{D}$ in $\boldsymbol{Z}$, or Egyptian fraction, with no loss of generality for common fraction (common fraction is given by Egyptian fraction multiplied by $\mathrm{N} \in \boldsymbol{Z}$, where $\mathrm{N}$ is the Numerator) is considered a simple integer division. In traditional rational representation, rational proper quotient is represented by infinite repetition of a basic digit cycle, called "reptend" (the repeating decimal part) [19]. The first repetition of basic digit cycle corresponds to the first full scale interval where number information can be conserved; CICT calls it "Representation Fundamental Domain" (RFD) [20]. In general, D, the denominator of the considered OSR (Outer Symbolic Representation) is given by a finite decimal word of length $\mathrm{W}_{\mathrm{D}}$ digits. From IOR (Inner OpeRational Representation) X, the related $\mathrm{RFD}_{\mathrm{L}}$ can be obtained, by a word length of $\mathrm{L}_{\mathrm{X}}$ digits. Elementary number theory considerations give us the usual worst case word length $\mathrm{L}_{\mathrm{X}}$ for $\mathrm{RFD}_{\mathrm{L}}$, with no loss of information, by:

$$
\mathrm{L}_{\mathrm{X}}=\mathrm{D}-1
$$

digits, if and only if 10 is a primitive root modulo $\mathrm{D}$. Otherwise Lx is a factor of (D-1). If the period of the repeating decimal of $1 / D$ for prime $D$ is equal $(D-1)$ then the repeating decimal part is called "cyclic number" and $\mathrm{D}$ can be referred as "primitive number" or "solid number" (SN) in CICT, or "full reptend prime" elsewhere [21]. Thus a $\mathrm{SN}$ is necessarily prime. It is a sufficient qualification, only. Conversely a prime number may not be a SN. So, the usual worst case word length $\mathrm{L}_{\mathrm{X}}$ for $\mathrm{X}$, given by eq.(1), can get $\mathrm{RFD}_{\mathrm{L}}$ with no loss of information, related to $\mathrm{D}$, just in case $\mathrm{D}$ is $\mathrm{SN}$. With no loss of generality, let us consider, the first Integer to show SN property manifestly, that is number $" \overline{0} 7 . \overline{0} "$. In this case $\mathrm{D}=7$, so that worst case length analysis gives $\mathrm{L}_{\mathrm{X}}=\mathrm{D}-1=6$ digits.

By realizing that the remainder $R_{1}$ is the fixed multiplicative ratio of a formal power series [06], the computation from generator $3^{\mathrm{n}}(\bmod 7)$ for $\mathrm{n}=1,2,3, \ldots$, till its exponential closure, gives the sequence the "Fundamental Cyclic Remainder Sequence" (FCRS):

$$
\mathrm{R}_{1}=3, \mathrm{R}_{2}=2, \mathrm{R}_{3}=6, \mathrm{R}_{4}=4, \mathrm{R}_{5}=5, \mathrm{R}_{6}=1 \text {, }
$$

from which the "Fundamental Cyclic Quotient Sequence" (FCQS) can be readily regenerated by $7 * \mathrm{R}_{\mathrm{n}}$ $(\bmod 10)$ :

$$
\mathrm{Q}_{1}=1, \mathrm{Q}_{2}=4, \mathrm{Q}_{3}=2, \mathrm{Q}_{4}=8, \mathrm{Q}_{5}=5, \mathrm{Q}_{6}=7 .
$$

So, quotient and remainder information can always be regenerated anew by remainder information only, but not vice-versa [20].

Therefore, 7 is just a SN and its full information content is usually captured by a six-digit word length $\mathrm{RFD}_{6}$, for $\mathrm{Q}_{6}$ and the same for $\mathrm{R}_{6}$, and the fullinformation content of long division $1 / 7$ would be stored into two decimal coupled words $<\mathrm{Q}, \mathrm{R}>$ having length $\mathrm{M}_{\mathrm{QR}}=(6+6)$ digits in total, for exact arbitrary precision computation. As a matter of fact, for all rational sequences, the Remainder $\mathrm{R}_{\mathrm{L}}$, at any computation evolutive stage $L_{X}$ (accuracy), is the fixed multiplicative ratio of a formal power series associated to the optimized decimal representations of their elementary generators [20]. Thus $1 / 7$ associated information content can be lossless compressed down to minimal $\mathrm{M}_{\mathrm{QR}}=(1+1)$ digits in total, in this specific case. As a matter of fact, any word couple $<\mathrm{Q}_{\mathrm{L}}, \mathrm{R}_{\mathrm{L}}>$ can be thought to be equivalent to and can represent a different real measurement instrument class, defined by RFD word length $\mathrm{L}_{\mathrm{X}}$. It is easy to see that, in general for $\mathrm{SN}$ of order $1\left(\mathrm{SN}_{1}\right.$ for short), the greater $\mathrm{D}$, the lengthier $\mathrm{L}_{\mathrm{X}}$, and higher the achievable compression ratio $\mathrm{E}_{\mathrm{w}}$.

In this simple case $\mathrm{E}_{\mathrm{w}}=(2 * 6) / 2=6: 1$, for number 7 . By this way, finite length coupled words can provide both finite arbitrary computational precision and relative precision exact error computability in a finite amount of time. By remainder knowledge, it is always possible to regenerate exact quotient and new remainder information at any arbitrary accuracy, with full information conservation. Thanks to the above arithmetic properties, the division algorithm can become free from trial and error like in finite segment $p$-adic representation system, but with no usually associated coding burden [21]. According to our humble knowledge, this is the first time that these arithmetic properties allow this kind of numeric awareness for arithmetic computational system. 
All the other members of the 1/7 RFD "Word Family Group" can be derived immediately, by cyclic permutation only, obtaining our result of Fig. 1. So, the final $\mathrm{SN}_{1}$ Family Group overall information compression ratio is given by $\mathrm{E}_{\mathrm{f}}=(2 * 6 * 6) / 2=36: 1$. It does not take a large leap in imagination to suspect that the next larger prime number $p$ to show SN property might be a good candidate for exhibiting cyclic-number property on an even larger scale. Unfortunately there does not seem to be any simple rule dictating which prime number $p$ will have $\mathrm{SN}_{1}$ property and which will not, so that one just has to check each prime out by long division to see. It turns out that the next higher prime number with the desired $\mathrm{SN}_{1}$ property is $p=\mathrm{D}=17$ where $\mathrm{T}_{p}=16$ digits. A search for still larger prime numbers with the same cyclic properties reveals that they are not at all rare. In fact no less than seven more prime numbers smaller than 100 generate cyclic numbers of order one or $\mathrm{SN}_{1}$. They are: $19,23,29,47,59,61$ and 97.

\begin{tabular}{|c|c|c|c|c|c|c|c|}
\hline $1 / 7$ & 0. & $\begin{array}{l}\mathrm{Q}_{1}=1 \\
\mathbf{R}_{1}=3\end{array}$ & $\begin{array}{l}\mathrm{Q}_{2}=4 \\
\mathrm{R}_{2}=2\end{array}$ & $\begin{array}{l}\mathrm{Q}_{3}=2 \\
\mathrm{R}_{3}=6\end{array}$ & $\begin{array}{l}\mathrm{Q}_{4}=8 \\
\mathrm{R}_{4}=4\end{array}$ & $\begin{array}{l}\mathrm{Q}_{5}=5 \\
\mathrm{R}_{5}=5\end{array}$ & $\begin{array}{l}\mathrm{Q}_{6}=7 \\
\mathrm{R}_{6}=1\end{array}$ \\
\hline $2 / 7$ & 0. & $\begin{array}{l}Q_{1}=2 \\
R_{1}=6\end{array}$ & $\begin{array}{l}\mathrm{Q}_{2}=8 \\
\mathrm{R}_{2}=4\end{array}$ & $\begin{array}{l}\mathrm{Q}_{3}=5 \\
\mathrm{R}_{3}=5\end{array}$ & $\begin{array}{l}\mathrm{Q}_{4}=7 \\
\mathrm{R}_{4}=1\end{array}$ & $\begin{array}{l}\mathbf{Q}_{5}=1 \\
\mathbf{R}_{5}=3\end{array}$ & $\begin{array}{l}\mathrm{Q}_{6}=4 \\
\mathrm{R}_{6}=2\end{array}$ \\
\hline $3 / 7$ & 0. & $\begin{array}{l}\mathrm{Q}_{1}=4 \\
\mathbf{R}_{1}=2\end{array}$ & $\begin{array}{l}\mathrm{Q}_{2}=2 \\
\mathrm{R}_{2}=6\end{array}$ & $\begin{array}{l}Q_{3}=8 \\
R_{3}=4\end{array}$ & $\begin{array}{l}\mathrm{Q}_{4}=5 \\
\mathrm{R}_{4}=5\end{array}$ & $\begin{array}{l}Q_{5}=7 \\
R_{5}=1\end{array}$ & $\begin{array}{l}\mathrm{Q}_{6}=1 \\
\mathrm{R}_{0}=3\end{array}$ \\
\hline $4 / 7$ & 0. & $\begin{array}{l}Q_{1}=5 \\
R_{1}=5\end{array}$ & $\begin{array}{l}\mathrm{Q}_{2}=7 \\
\mathrm{R}_{2}=1\end{array}$ & $\begin{array}{l}\mathrm{Q}_{3}=1 \\
\mathrm{R}_{3}=3\end{array}$ & $\begin{array}{l}\mathrm{Q}_{4}=4 \\
\mathrm{R}_{4}=2\end{array}$ & $\begin{array}{l}\mathrm{Q}_{5}=2 \\
\mathrm{R}_{5}=6\end{array}$ & $\begin{array}{l}\mathrm{Q}_{6}=8 \\
\mathrm{R}_{6}=4\end{array}$ \\
\hline $5 / 7$ & 0. & $\begin{array}{l}Q_{1}=7 \\
\mathbf{R}_{1}-1\end{array}$ & $\begin{array}{l}\mathrm{Q}_{2}=1 \\
\mathrm{R}_{2}-3\end{array}$ & $\begin{array}{l}\mathrm{Q}_{3}=4 \\
\mathrm{R}_{3}-2\end{array}$ & $\begin{array}{l}\mathrm{Q}_{4}=2 \\
\mathrm{R}_{4}-6\end{array}$ & $\begin{array}{l}Q_{5}=8 \\
R_{5}-4\end{array}$ & $\begin{array}{l}Q_{6}=5 \\
\mathrm{R}_{6}-5\end{array}$ \\
\hline $6 / 7$ & 0. & $\begin{array}{l}Q_{1}=8 \\
R_{1}=4\end{array}$ & $\begin{array}{l}\mathrm{Q}_{2}=5 \\
\mathrm{R}_{2}=5\end{array}$ & $\begin{array}{l}\mathrm{Q}_{3}=7 \\
\mathrm{R}_{3}=1\end{array}$ & $\begin{array}{l}\mathrm{Q}_{4}=1 \\
\mathrm{R}_{4}=3\end{array}$ & $\begin{array}{l}\mathrm{Q}_{5}=4 \\
\mathrm{R}_{5}=2\end{array}$ & $\begin{array}{l}\mathrm{Q}_{6}=2 \\
\mathrm{R}_{6}=6\end{array}$ \\
\hline $7 / 77$ & 0. & $\begin{array}{l}\mathrm{Q}_{1}=9 \\
\mathrm{R}_{1}=7\end{array}$ & $\begin{array}{l}\mathrm{Q}_{2}=9 \\
\mathrm{R}_{2}=7\end{array}$ & $\begin{array}{l}\mathrm{Q}_{3}=9 \\
\mathrm{R}_{3}=7\end{array}$ & $\begin{array}{l}\mathrm{Q}_{4}=9 \\
\mathrm{R}_{4}=7\end{array}$ & $\begin{array}{l}\mathrm{Q}_{5}=9 \\
\mathrm{R}_{5}=7\end{array}$ & $\begin{array}{l}\mathrm{Q}_{6}=9 \\
\mathrm{R}_{6}=7\end{array}$ \\
\hline
\end{tabular}

Fig. 1. $Q_{n}$ and $R_{n}$ values for each component of $\mathrm{SN}_{1}$ Word Family Group 1/7 [06].

Till now we discussed a peculiar number property focusing our attention, for simplicity of presentation, on $\mathrm{SN}_{1}$ only, but it does not imply that a number has to be a $\mathrm{SN}_{1}$ in order to generate intriguing cycles. For instance, Family Group 1/13 contains the smallest cyclic-numbers of order two or $\mathrm{SN}_{2}$. What about more second order cyclics? The basic ones are also generated by a subset of prime fractions $1 / p$. The other prime numbers smaller than 100 which generate second order cyclics are 31,43 , $67,71,83$ and 89 . In the same manner one can now go on to define, and find, cyclic numbers of order three, four, five, and higher and higher, according to his own computational needs. The smallest prime number producing the Family Group of third order or $\mathrm{SN}_{3}$ cyclicnumber is 103 . In this case, $\mathrm{T}_{p}=34$ digit string (so we have $3 * 34=102$, instead of the unique 102 digit string a first order cyclic would have). The seven smallest primes generating cycles of order four through ten are respectively $53,11,79,211,41,73$, and 281 , but you must reach prime number 353 to get the first prime generating cycle of order 11 or $\mathrm{SN}_{11}$. All the prime numbers less than 100 have now been covered with the exception of $p=37$, with $\mathrm{T}_{p}=3$ digits. Therefore 37 is a cyclic number of order 12 or $\mathrm{SN}_{12}$. Multiplication composition of prime numbers can generate an entire universe of new number-cycles. So any modular group can be better represented by generators and relations. One of the earliest presentations of a group by generators and relations was given by the Irish mathematician William Rowan Hamilton in 1856, in his Icosian Calculus, a presentation of the icosahedral group [22], [23]. The first systematic study was given by German mathematician Walther Franz Anton von Dyck (18561934) [24], student of German mathematician Christian Felix Klein (1849-1925), in the early 1880s, laying the foundations for combinatorial group theory [25]. Every group has a presentation, and in fact many different presentations; a presentation is often the most compact way of describing the structure of the group. In abstract algebra, the "fundamental theorem of cyclic groups" states that every subgroup of a cyclic group $G$ is cyclic. Moreover, the order " $\mathrm{k}$ " of any subgroup of a cyclic group $G$ of order $n$ is a divisor of $n$, and for each positive divisor "k" of $n$, the group $G$ has exactly one subgroup of order "k". This is just the first step to start an intriguing voyage from the concept of "presentation of a group" to the concept of "representation theory" for combinatorial modular group theory [26]. Traditional rational number system $\boldsymbol{Q}$ can be regarded as a highly sophisticated open logic, powerful and flexible formal language, with selfdefining consistent words and rules, starting from elementary generators and relations [20].

\section{Application example}

The rich operative scenario offered by combinatorial modular group theory is full of articulated solutions to information processing problems.

Table 3. Picture benchmark database list [27].

\begin{tabular}{|c|c|c|c|c|}
\hline N. & NAME & PIXELS & SIZE (MB) & BPP \\
\hline 01 & artificial & $3072 \times 2048$ & 36.80 & 24 \\
\hline 02 & big_building & $7216 \times 5412$ & 223.36 & 24 \\
\hline 03 & big_tree & $6088 \times 4550$ & 158.50 & 24 \\
\hline 04 & cathedral & $2000 \times 3008$ & 34.42 & 24 \\
\hline 05 & fireworks & $3136 \times 2352$ & 42.21 & 24 \\
\hline 06 & flower_foveon & $2268 \times 1512$ & 19.62 & 24 \\
\hline 07 & hdr & $3072 \times 2048$ & 36.80 & 24 \\
\hline 08 & leaves_iso_1600 & $3008 \times 2000$ & 34.42 & 24 \\
\hline 09 & leaves_iso_200 & $3008 \times 2000$ & 34.42 & 24 \\
\hline 10 & nightshot_iso_100 & $3136 \times 2352$ & 42.29 & 24 \\
\hline 11 & nightshot_iso_1600 & $3136 \times 2352$ & 42.29 & 24 \\
\hline 12 & spider_web & $4256 \times 2848$ & 69.36 & 24 \\
\hline
\end{tabular}


For instance, Word Family Group $\mathrm{SN}_{1}$, discussed in the previous section, shows peculiar, cyclical properties that can be conveniently used to get interesting image lossless comp/decomp algorithm. Please, note from Fig.1 that remainder $\mathrm{R}_{\mathrm{n}}=a=1,2, \ldots, p-1$, can be thought as pointer to $\mathrm{Q}_{\mathrm{n}}+1$ to get the beginning of $a / \mathrm{D}$ quotient cyclical string immediately, with no computation at all, but the initial one for $\mathrm{SN}_{1}$ Word Family $p$. Combinatorial optimization is achieved by finding the best $\mathrm{SN}_{1}=p$ which allow to minimize a desired constraint within a specified interval for each image. Then, $a / p$ will be best in that no other rational in that specified interval will have a smaller numerator or a smaller denominator. To check our idea, we used an Internet public picture database for 24 bpp color images [27] as reported by Table 3 and Fig.2. Twelve original colour pictures with different dynamic range and different overall content were considered. They are lossless compressed from 24 to 16 bpp by JPEG-LS (Lossless JPEG), JPEG 2000, HD Photo (JPEG XR) algorithms and by ours that is called UC (Universal Compression).

\subsection{Lossless compression technical details}

Lossless JPEG was developed as a late addition to JPEG in 1993, using a completely different technique from the lossy JPEG standard developed previously. It uses a predictive scheme based on the three nearest (causal) neighbors (upper, left, and upper-left), and entropy coding is used on the prediction error.

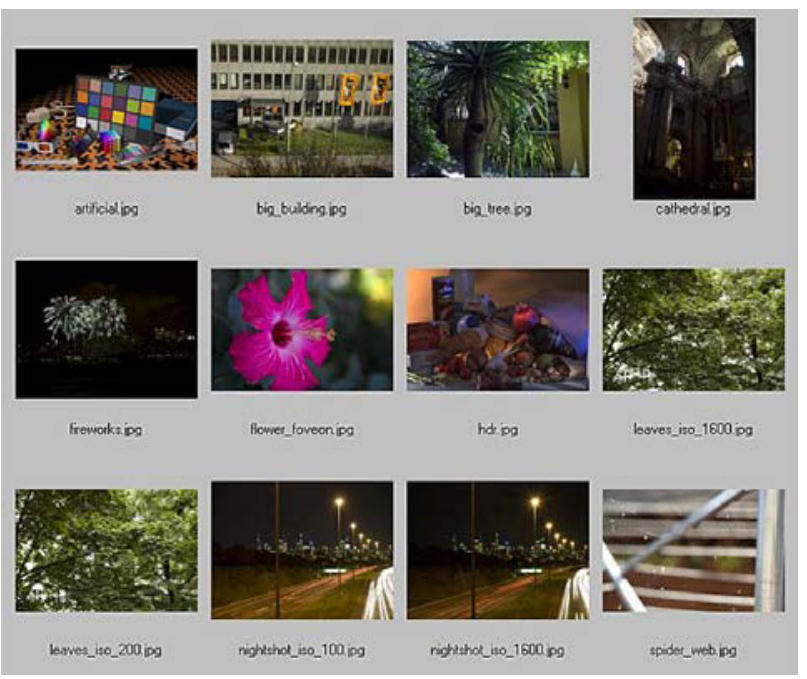

Fig. 2. Pictures in Benchmark Database as from Table 1, for our application example [27].

The standard Independent JPEG Group libraries cannot encode or decode it, but Ken Murchison of Oceana Matrix Ltd. wrote a patch that extends the IJG library to handle Lossless JPEG. It has some popularity in medical imaging, and is used in DNG (Adobe) and some digital cameras to compress raw images, but otherwise was never widely adopted. It might be used as an umbrella term to refer to all lossless compression schemes developed by the Joint Photographic Expert group. They include JPEG 2000 and JPEG-LS.
JPEG-LS is a lossless/near-lossless compression standard for continuous-tone images. Its official designation is ISO-14495-1/ITU-T.87. It is a simple and efficient baseline algorithm which consists of two independent and distinct stages called modeling and encoding. It was developed with the aim of providing a low-complexity lossless and near-lossless image compression standard that could offer better compression efficiency than JPEG. At the time, the Huffman codingbased JPEG lossless standard and other standards were limited in their compression performance. Total decorrelation cannot be achieved by first order entropy of the prediction residuals employed by these inferior standards. JPEG-LS, on the other hand, can obtain good decorrelation. Part 1 of this standard was finalized in 1999. Part 2, released in 2003, introduced extensions such as arithmetic coding. The core of JPEG-LS is based on the LOCO-I algorithm [28] that relies on prediction, residual modeling and context-based coding of the residuals. Most of the low complexity of this technique comes from the assumption that prediction residuals follow a two-sided geometric distribution (also called a discrete Laplace distribution) and from the use of Golomb-like codes, which are known to be approximately optimal for geometric distributions. Besides lossless compression, it also provides a lossy mode ("near-lossless") where the maximum absolute error can be controlled by the encoder. Compression for JPEG-LS is generally faster than JPEG 2000 and much better than the original lossless JPEG standard. Here JPEG-LS is used for historical reason comparison, JPEG 2000 provides lossless compressions for images with bit depth no more than 16 bit per channel, and allows progressive transmission with signal-to-noise ratio (SNR) and spatial scalability. In particular, it supports ROI (Region Of Interest) coding, so that image regions with diagnostic importance can have much higher quality than the other parts. The codestream obtained after compression of an image with JPEG 2000 is downscalable in nature, meaning that it can be decoded in a number of ways; for instance, by truncating the codestream at any point, one may obtain a representation of the image at a lower resolution, or SNR. However, as a consequence of this flexibility, JPEG 2000 requires encoders/decoders that are complex and computationally demanding.

HD Photo slgorithm is based on technology originally developed and patented by Microsoft. It supports deep color images with 48-bit RGB, both lossy and lossless compression, and is the preferred image format for Ecma-388 Open XML Paper Specification documents. HD Photo offers several major key improvements over JPEG, including better compression, lossless compression, tile structured support, more color accuracy, transparency map and metadata support. HD Photo is conceptually very similar to JPEG: the source image is optionally converted to a luma-chroma colorspace, the chroma planes are optionally subsampled, each plane is divided into fixed-size blocks, the blocks are transformed into the frequency domain, and the frequency coefficients are quantized and entropy coded. In July 2007, the Joint Photographic Experts 
Group and Microsoft announced HD Photo to be under consideration to become a JPEG standard known as JPEG XR. On 16 March 2009, JPEG XR was given final approval as ITU-T Recommendation T.832 and starting in April 2009, it became available from the ITU-T in "pre-published" form. On 19 June 2009, it passed an ISO/IEC Final Draft International Standard (FDIS) ballot, resulting in final approval as International Standard ISO/IEC 29199-2. The ITU-T updated its publication with a corrigendum approved in December 2009, and ISO/IEC issued a new edition with similar corrections on 30 September 2010. In 2010, after completion of the image coding specification, the ITU-T and ISO/IEC also published a motion format specification (ITU-T T.833|ISO/IEC 29199-3), a conformance test set (ITU-T T.834|ISO/IEC 29199-4), and reference software (ITU-T T.835|ISO/IEC 29199-5) for JPEG XR. In 2011, they published a technical report describing the workflow architecture for the use of JPEG XR images in applications (ITU-T T.Sup2|ISO/IEC TR 29199-1). In April 2013, Microsoft released an open source JPEG XR library under the BSD licence. As of August 2014, there were still no cameras that shoot photos in the Jpeg XR (.JXR) format.

UC provides lossless compressions for images with arbitrary bit depth (ABD) per channel, and allows progressive transmission with SNR and spatial scalability, at no extra cost. Specifically, it supports ROI (Region Of Interest) coding, so that image regions with diagnostic importance can have much higher quality than the other parts. The codestream obtained after compression of an image with UC is downscalable and upscalable in nature, meaning that it can be decoded in a number of ways; for instance, by truncating the codestream at any point, one may obtain a representation of the image at a lower resolution, or signal-to-noise ratio. Different from all other algorithms, this time, if you have local computational resources, you can regenerate the original information arbitrarily to higher BD. As a consequence of this flexibility, you do have to pay no extra cost!

\subsection{Results and discussion}

Compression results for 24 to $16 \mathrm{bpp}$ lossless compression of the twelve database pictures reported in Table 3, with different content and different extended dynamic each, show that, on average, UC takes about $17 \%$ more space than JPEG 2000. Nevertheless UC shows compression speed about $17 \%$ faster than JPEG 2000 on average. In order to achieve an overall evaluation and a fair comparison between so different algorithms, we define the key performance index (KPI) given by compressed bit-per-pixel (bpp) times compression time ( $\mathrm{s}$, in second) for each picture, where:

$$
\mathrm{KPI}=\operatorname{bpp} \times \mathrm{s}
$$

Our first raw results, with no further algorithm refinement out of cyclic family computation only, as described in Section 3.2, show that overall UC KPI compare quite well to HD Photo and it is better than traditional JPEG 2000, with no further algorithmic development effort or computational load.

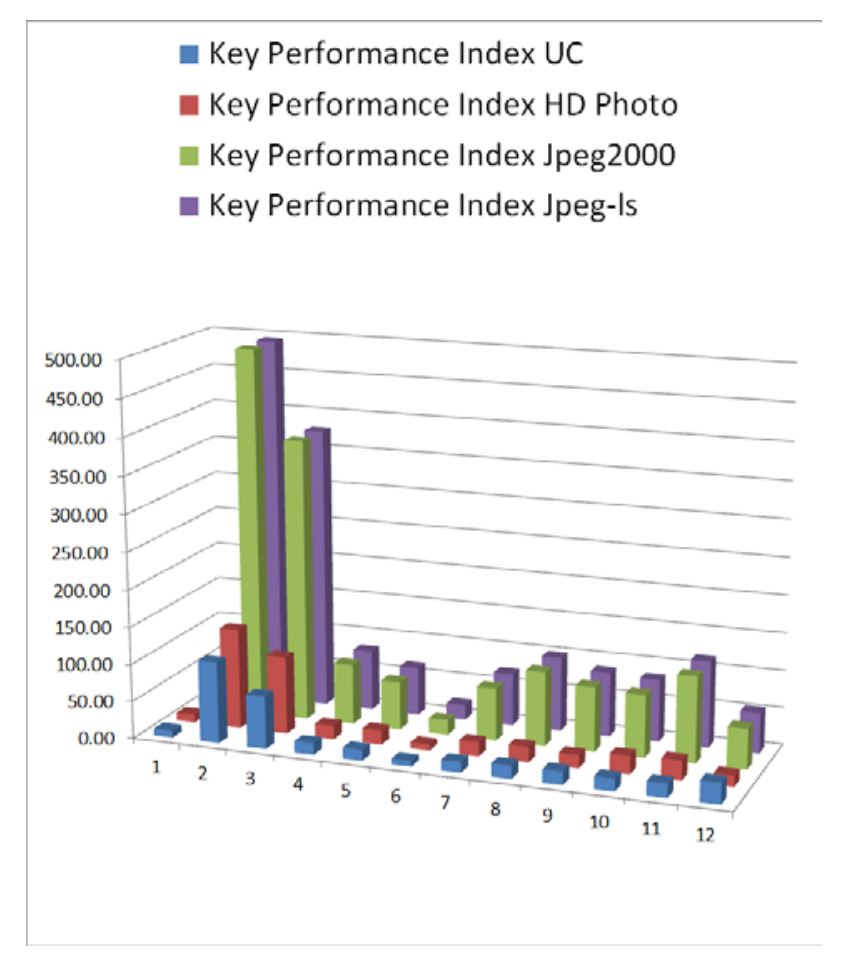

Fig. 3. KPI 24 to 16 bpp lossless compression result for JPEGLS, JPEG 2000, HD Photo and UC algorithms (from back to front respectively): ordinate in KPI units, abscissa number ordering refers to picture list as from Table 1.

The only image where UC KPI is greater than HD Photo KPI is picture number 12, the "spider_web" picture shown in Fig.4. As you can see from that picture, different from all other picture in our Benchmark Database, there are a lot of linear, finer details with delicate superpositions embedded in it and HD Photo algorithm can take advantage from them quite easily.

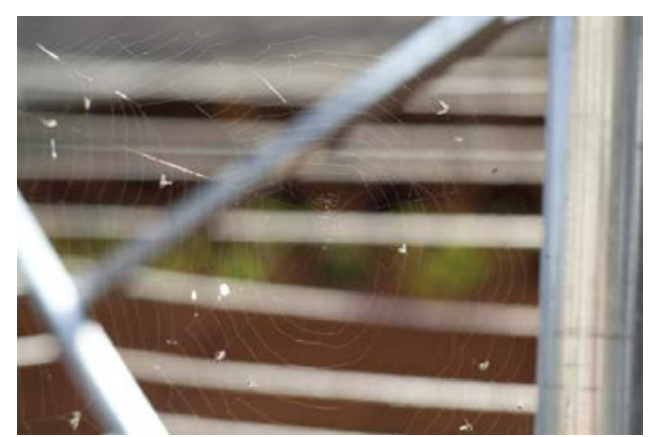

Fig. 4. Spider_web picture as n.12 test picture in our Picture Benchmark Database.

$\mathrm{UC}$ is a zero-knowledge universal lossless compression algorithm that does not use linear a priori knowledge to achieve better result. Therefore, for general purpose application raw UC on average can perform better than HD Photo, but for pictures full of superposed linear, finer details it cannot compete with HD Photo. Nevertheless, raw UC algorithm parameters have not yet 
been quite tuned for overall optimum performance, so there is still room for further improvement. UC exploits arbitrary bit depth (ABD) per channel and full information conservation and regeneration by design. UC method differs from the existing ones in three main features. First, it is not a traditional extended bit depth encoding/decoding and does not use Gray-Golomb coding to support progressive transmission as in VOI (Value Of Interest) approaches [29].

Second, UC scheme can utilize dynamic BD settings for original images to support dynamic BD scalability, which allows pixels to be progressively reconstructed in the order according to their display importance. Pixel reconstruction can be centralized or decentralized, local or global. Third, we can consider end-user display computational resource availability, for end-point information regeneration and network bandwidth minimization. Different from all other algorithms, not only a downscaling, but even an upscaling is possible by UC information conservation and regeneration. For images with extended bit-depth, the pixel values can be displayed after BD mapping to fit the display bit depth. Thus only pixels with interested values can be visually important, under certain predefined BD parameters.

\section{Conclusion}

We have presented results on an universal zeroknowledge combinatorial lossless compression scheme called UC, for extended dynamic range images, with arbitrary bit depth, based on information conservation and regeneration according to $C I C T$ by design. Its raw overall zero-knowledge lossless compression performance compare quite well to standard ones, but offering true $\mathrm{ABD}$ and achieving full image information conservation and regeneration, at no extra cost, without complex and computationally demanding encoders/decoders.

\section{References}

1. N. Reims, T. Schoen, M. Boehnel, F. Sukowski, M. Firsching, Proc. of SPIE "Optics + Photonics", Soc. Photo-Opt. Instr. Eng., 921209 (2014).

2. K.J. Batenburg, J. Sijbers, IEEE Trans. Image Process. 20(9), 2542 (2011).

3. K.J. Batenburg, S. Bals, J. Sijbers, C. Kübel, P.A. Midgley, J.C. Hernandez, U. Kaiser, E.R. Encina, E.A. Coronado, G. Van Tendeloo, Ultramicr. 109, 730 (2009).

4. M.H. Kryder, S.K. Chang, IEEE Trans. Mag. 45(10) (October 2009). Available at: $<\mathrm{URL}=$

http://isites.harvard.edu/fs/docs/icb.topic1195323.files/A fter_Hard_Drives.pdf $>$.

5. D.A. Koff, H. Shulman, Can. assoc. of radiologists jour. 57, 211 (2006).

6. M.J. Zukoski, T. Boult, T. Iyriboz, Int. J. of Bioinf. Research and Applications 2(1), 89 (2006).

7. T.J. Kim, K.H. Lee, B. Kim, E.J. Chun, V. Baipal, Y.H. Kim, S. Hahn, K.W. Lee, Eur. J. Radiol. 69(3), 483 (2009).
8. The DICOM standard, (2009). Available at: $<\mathrm{URL}=$ http://dicom.nema.org/dicom>.

9. ISO/IEC 15444 (2007). Available at: $<\mathrm{URL}=$ https://www.iso.org/standard/41570.html >.

10. Wikipedia, Jpeg2000 (2017). Available at: $<\mathrm{URL}=$ https://en.wikipedia.org/wiki/JPEG 2000>.

11. C.E. Shannon, Bell Syst. Tech. J. 27(3) 379 (July/October 1948).

12. A. Schrijver, (2017). Available at: $<\mathrm{URL}=$ http://homepages.cwi.nl/ lex/files/dict.pdf $>$.

13. K.L. Hoffman, T.K. Ralphs, (2012). Available at: $<\mathrm{URL}=$ http://coral.ie.lehigh.edu/ ted/files/papers/I CO-EORMS11.pdf $>$.

14. L. Fortnow, Comm. ACM 52(9), 78 (2009).

15. R.A. Fiorini, in N.E. Mastorakis, M. Demiralp, N. Mukhopadhyay, F. Mainardi, Eds., Advances in Applied mathematics, Modelling and Simulation, Mathematical and Computers in Science and Engineering Series $n .34$ (Florence, Italy, WSEAS Press, 385-394, 2014).

16. R.A. Fiorini, Intern. J. Syst. Applications, Eng. and Dev. 9, 93 (2015). Available at: $<\mathrm{URL}=$

http://www.naun.org/main/UPress/saed/2015/a305994003.p $\mathrm{df}>$.

17. R.A. Fiorini, Fundam. Inform. 135(1-2),135 (2014).

18. R.A. Fiorini, G. Laguteta, in V. Mladenov, Ed., Proceedings of the 19th International Conference on Systems (Zakynthos Island, Greece, 648-655, 2015).

19. E.W. Weisstein, (1999-2012). Available at: $<\mathrm{URL}=$ http://mathworld.wolfram.com/FullReptendPrime.html $>$.

20. R.A. Fiorini, G. Laguteta, Fundam. Inform. 125(3-4), 261 (2013).

21. D.M. Young, R.T. Gregory, A Survey of Numerical Mathematics, vol.I and II (Reading, Mass.: Addison Wesley, 1973).

22. W.R. Hamilton, Phil. Mag. 12, 446 (1856).

23. B. Chandler, W. Magnus, The History of Combinatorial Group Theory: A Case Study in the History of Ideas, Studies in the History of Mathematics and Physical Sciences (1st ed., Springer, 234, December 1, 1982).

24. W. von Dyck, Math. Ann. 20(1), 1 (1882).

25. J. Stillwell, Mathematics and its history (Springer 2002).

26. C.W. Curtis, Pioneers of Representation Theory: Frobenius, Burnside, Schur, and Brauer, History of Mathematics (Providence, R.I.: American Mathematical Society, 2003).

27 The New Test Images for Image Compression Benchmark (Jan 2008). Available at: $<\mathrm{URL}=$

http://www.imagecompression.info/test images $>$.

28. M. Weinberger, G. Seroussi, G. Sapiro, IEEE Trans. Im. Proc. 9(8), 1309 (Aug. 2000). Available at: $<\mathrm{URL}=$

http://www.labs.hp.com/research/info theory/loco/>.

$<\mathrm{URL}=$

http://www.labs.hp.com/research/info theory/loco/HPL-98193R1.pdf $>$.

29. W. Sun, Y. Lu, W. Feng, L. Shipeng, IEEE, ICIP, 1749, (2009). Available at: $<$ URL:

http://toc.proceedings.com/07230webtoc.pdf >. 